\title{
Determinants of healthy ageing: a systematic review of contemporary literature
}

\author{
Thais Abud $^{1} \cdot$ Georgios Kounidas ${ }^{1}$ (D) $\cdot$ Kathryn R. Martin $^{2} \cdot$ Martin Werth $^{3} \cdot$ Kay Cooper $^{4} \cdot$ Phyo Kyaw Myint $^{1}$
}

Received: 26 October 2021 / Accepted: 5 December 2021 / Published online: 8 February 2022

(c) The Author(s), under exclusive licence to Springer Nature Switzerland AG 2022

\begin{abstract}
Background Healthy ageing frameworks have been highly explored. Our objective was to assess existing frameworks for healthy ageing and to identify commonly described factors that can potentially act as determinants of healthy ageing.

Methods We carried out a systematic review by searching five electronic databases (EMBASE, MEDLINE, Cochrane, PsychINFO, and CINAHL) from January 2010 to November 2020 to capture contemporary evidence. Eligible studies needed to report a clear framework of healthy ageing in humans, within one or more of three domains (physical, mental/cognitive, social), in English. No restriction was placed on geographical location. Retrospective studies, studies that did not report a framework of healthy ageing, and studies with a focus on diagnostic measures were excluded.

Results Of 3329 identified records, nine studies met our eligibility criteria and were included. Most of the studies were qualitative or cross sectional, and a majority were carried out in Asia, followed by North America, Australia, and Africa. The ten determinants identified for healthy ageing include physical activity, diet, self-awareness, outlook/attitude, life-long learning, faith, social support, financial security, community engagement, and independence.

Conclusions We identified ten determinants of healthy ageing proposed by the contemporary evidence base. There appears to be increasing acknowledgement of the instrumental role of social and mental/cognitive well-being as determinants of healthy ageing. The extent to which each determinant contributes to healthy ageing requires further evaluation.
\end{abstract}

Keywords Healthy ageing $\cdot$ Determinants $\cdot$ Framework

\section{Introduction}

Worldwide, the population aged over 65 is increasing at a faster pace than all other age groups [1]. As a result of this demographic shift, it is important to look at ways to improve the quality of life of older adults and support independent living. The COVID-19 pandemic has

Thais Abud and Georgios Kounidas are joint first authors.

Phyo Kyaw Myint

phyo.myint@abdn.ac.uk

1 School of Medicine, Medical Sciences and Nutrition, University of Aberdeen, Aberdeen AB25 2ZD, UK

2 Academic Primary Care, Institute of Applied Health Sciences, Aberdeen Centre for Arthritis and Musculoskeletal Health, University of Aberdeen, Aberdeen AB25 2ZD, UK

3 Autumn Project Limited, London SE27 0BY, UK

4 School of Health Sciences, Robert Gordon University, Aberdeen AB10 7QG, UK disproportionately affected people over 65 years of age, who had previously been in good health [2]. Given the global impact of COVID-19, it is more crucial than ever to identify determinants of healthy ageing that can be applicable across different communities and countries to build their path to better health.

Ageing as a concept has been vastly explored, a particularly important aspect being how to define what it means to age well. Key leaders in the field of ageing, such as Rowe and Kahn, defined successful ageing as the absence of physical impairment and chronic diseases, as well as optimal social participation and mental well-being [3]. Rowe and Kahn brought the field forward with their inclusion of mental and social well-being. The idea that to age healthily one must be free of disease or impairment is something that has been carried throughout the years, but in more contemporary times this has been disputed and modified.

Previous reviews in this field have provided valuable information on internal and external factors that promote 
healthy ageing in older age, as well as better engagement in healthier and active lifestyles [4, 5]. In 2013 Lara et al. developed five fundamental domains of healthy ageing: physiological and metabolic health; physical capability; cognitive function; social well-being, and psychological well-being [6]. Comparatively in 2017 Hornby-Turner et al. categorised four domains: personal, social, economic, and environmental [4]. This shows the lack of consensus of what ageing well entails due to the variability between studies.

Lu et al., a review comparing methods used to assess healthy ageing, evaluated the common terms used in ageing studies (e.g. successful ageing, active ageing), and established that the term healthy ageing was most appropriate for their study [7]. The main reason as to why healthy was preferred was because of the World Health Organization's (WHO) definition. The WHO defines health as "a state of complete physical, mental/cognitive, and social well-being, rather than merely the absence of disease or infirmity" [8]. The WHO established their definition of health in their constitution in 1948 and still stand by the initial definition. It highlights that being healthy is not solely determined by the absence of disease, even though may be a contributor. The WHO's definition also highlights the three main domains of health: physical, mental, and social well-being [8]. Separating healthy ageing into these three domains can facilitate the development of a framework to assess and guide an individual towards healthy ageing.

The aim of this systematic review was to synthesise the evidence on healthy ageing frameworks by critically evaluating existing frameworks, identifying the methods used in frameworks to evaluate health ageing, and if appropriate to propose a revised, contemporary framework for healthy ageing. In doing so also to identify factors that can act as determinants of healthy ageing within the domains of physical, mental/cognitive, and social well-being in line with the WHO definition of health [8].

\section{Methods}

We carried out a systematic literature review by searching five databases [EMBASE (Ovid), MEDLINE (Ovid), Cochrane Central Register of Controlled Trials (Ovid), PsychINFO (Ovid), CINAHL (EBSCO)] in November 2020, in accordance with the Preferred Reporting Items for Systematic Reviews and Meta-Analysis (PRISMA) statement [9]. The PRISMA checklist was included in the supplementary material, as in Table 1. A PRISMA protocol was not registered.

\section{Search strategy}

The following search terms were used in OVID (EMBASE, MEDLINE, Cochrane, PsychINFO): healthy ageing.mh. or (healthy ageing or healthy ageing).tx,tw,ab,hw,kw.) and (measurement tool or scale or instrument or questionnaire). mp. and EBSCOhost (CINAHL): MH(healthy ageing) OR TX(healthy ageing OR healthy ageing) AND (measurement tool OR scale OR instrument OR questionnaire).

\section{Eligibility}

To be eligible for this systematic review, studies were required to meet the following criteria: (1) Studies published in English, (2) Articles published between January 2010 and November 2020 (to capture contemporary evidence), and (3) Studies that were conducted in humans. There were no restrictions for inclusion based on geographical location. The following exclusion criteria were applied: (1) Retrospective studies, (2) Studies that did not report a framework of healthy ageing, and (3) Studies with a focus on clinical diagnostic measures (e.g. Magnetic Resonance Imaging (MRI)).

\section{Study identification}

All identified studies were transferred to Covidence (Melbourne, Australia) systematic review software where they were deduplicated [10]. The titles and abstracts were screened by two independent reviewers (GK, TA) with conflicts resolved by discussion or a third reviewer (PKM). Following that, full-text screening was conducted on all retrieved studies by two independent reviewers, with conflicts similarly resolved by discussion or a third reviewer (PKM). Reasons for exclusion at full-text screening stage are reported in the PRISMA flow chart (Fig. 1).

\section{Outcomes and data extraction}

The main outcome was a framework for successful healthy ageing. For this systematic review, outcomes also included identification of determinants that fall within the three domains of physical, mental/cognitive, and social wellbeing. Data were independently extracted from included studies by two reviewers (TA, GK). Disagreement was resolved by discussion and/or by a senior author (PKM). The following data were extracted: country, study design, age, number of participants, gender, specific population studied, main framework, and healthy ageing domains. 


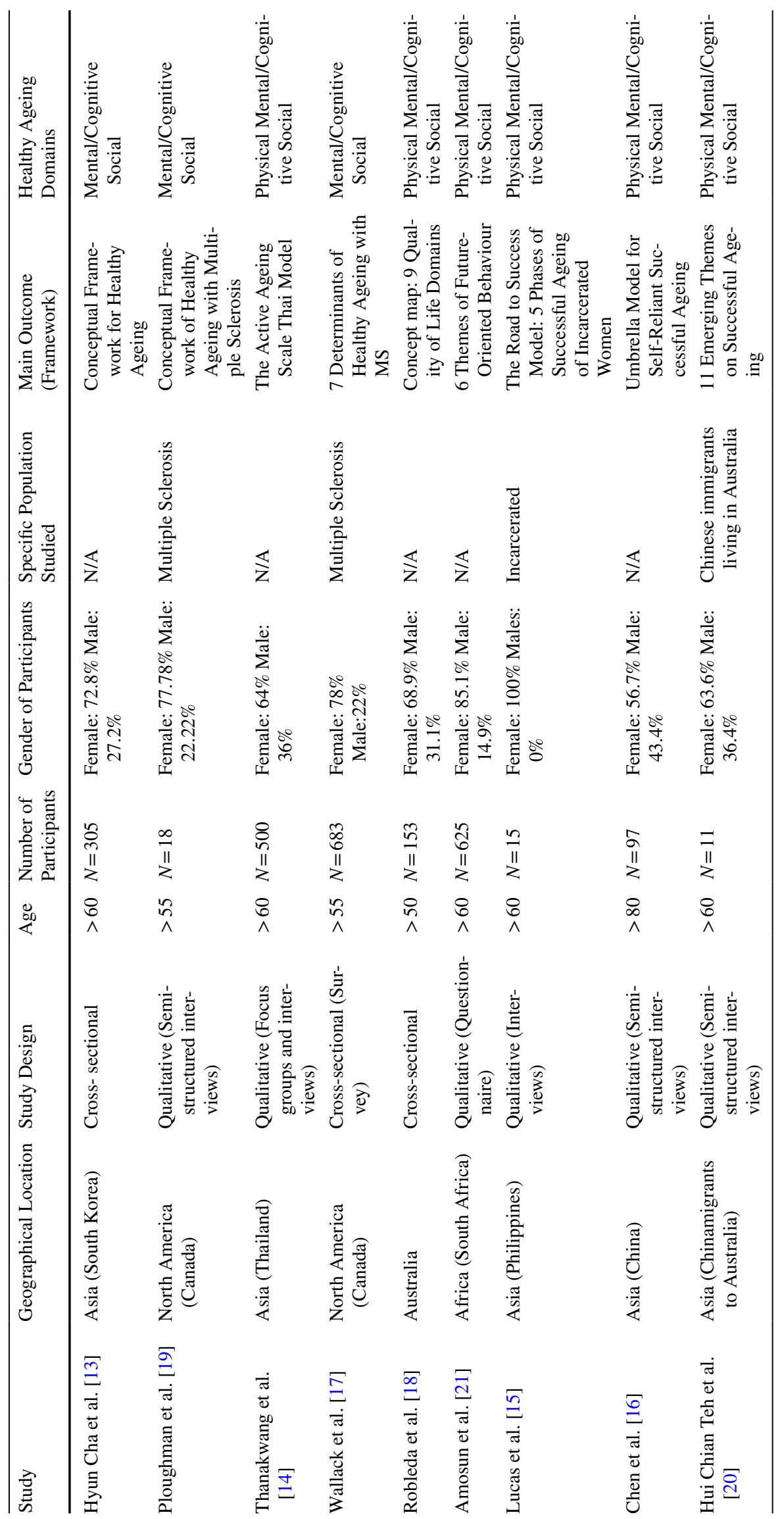




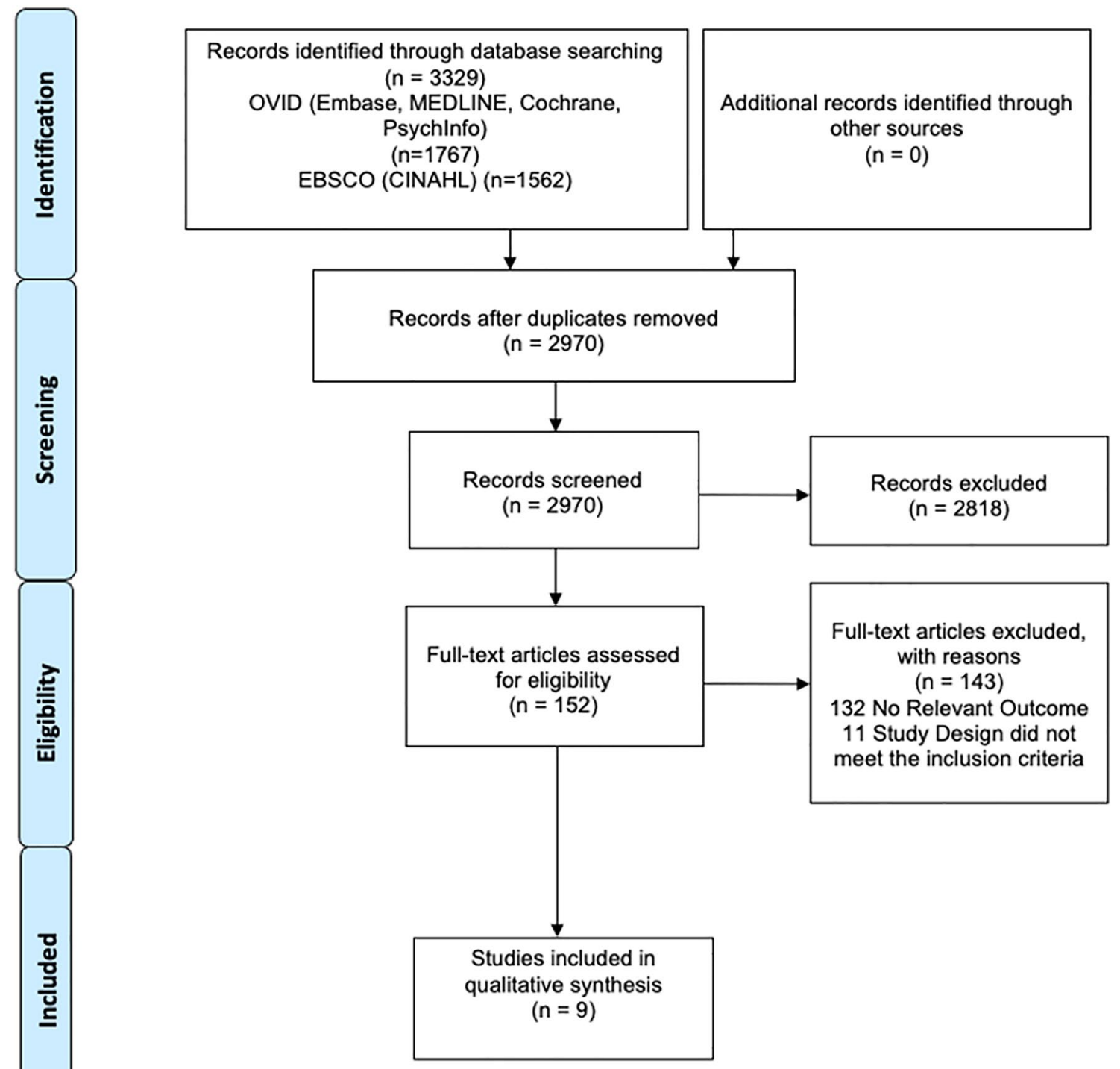

Fig. 1 PRISMA 2009 Flow Diagram

\section{Derived frameworks and categorisation into domains}

Following full-text screening and data extraction, due to the nature of studies, meta-analysis was not feasible; therefore, we conducted a narrative synthesis. A framework for healthy ageing was identified as a primary outcome in all included studies (Supplementary material).

\section{Quality assessment}

Included studies were critically appraised independently by two researchers (TA, GK), using the Critical Appraisal Skills Programme (CASP) Checklist for qualitative studies and the
Newcastle-Ottawa Quality Assessment Scale (NOS) adapted for cross-sectional studies $[11,12]$.

\section{Results}

\section{Study selection}

Of 3329 studies initially identified, after removing duplicates, 2970 studies underwent title/abstract screening during which 2818 studies were excluded for the following reasons: did not focus on healthy ageing and/or had a focus on diagnostic measures (e.g. MRI). Thus, a total of 152 studies were retrieved in full and screened against the inclusion and exclusion criteria by two reviewers 
independently (GK, TA) to determine their eligibility; 143 studies were excluded, as they did not report a framework for healthy ageing. Nine studies that reported frameworks of healthy ageing were included in the review (Fig. 1) [13-21].

\section{Quality assessment}

All studies were found to be of high quality according to the CASP Checklist for qualitative studies and the NOS for cross-sectional studies (Supplementary Table 2, Supplementary Table 3). Five qualitative studies did not adequately report the relationship between the researcher and the participants [14-21]. Two cross-sectional studies did not report the comparability between respondents and non-respondents $[13,18]$.

\section{Study characteristics}

The total number of participants in this review was of 2407 , ranging from 11 to 683 participants in individual studies (Table 1). Most studies had a sample size greater than 100, and were predominantly conducted in Asia [13-16]. Eight studies were carried out on both genders and one was solely on females. A majority of participants were above sixty years of age: study mean ages ranged from 64 to 85.2. Most of the studies were qualitative in nature and employed either semi-structured interviews or focus groups. Three studies used cross-sectional design (e.g. surveys) [13, 17, 18]. There were four studies that were conducted in people with specific conditions or circumstances. Two focused on Multiple
Sclerosis (MS) patients [17, 19], one on incarcerated women [15], and one on immigrants [20].

\section{Determinants of healthy ageing}

\section{Overview}

Six out of the nine studies included determinants of successful ageing within the three healthy ageing domains of physical, mental/cognitive, and social well-being (Table 2, Fig. 2) $[14-17,20,21]$. Three studies only addressed the mental/ cognitive and social domains. Of the nine studies, there were five that had determinants that covered more than a single domain, meaning the determinant could not be solely classified into one domain $[14,15,17,18,20]$. Ten overall determinants were identified, with independence being present in all three domains. Figure 2 shows the combination of determinants found in each study by the overlapping of the shapes, each of which represents a study.

\section{Physical well-being}

Seven studies included determinants within the physical domain [14-18, 20,21]. These studies emphasised the need to maintain a good level of physical capability to enhance successful healthy ageing. Wallack et al. focused on MS participants; therefore, physical activity was addressed as a subtype of "lifestyle choices and habits" specifically in the body category [27]. This included exercise but also alternative therapies and medication management due to their potential effects on the body. Conversely, the other studies

Table 2 Determinants of healthy ageing

\begin{tabular}{|c|c|c|c|c|c|c|c|c|c|}
\hline Studies & $\begin{array}{l}\text { Hyun Cha } \\
\text { et al. [13] }\end{array}$ & $\begin{array}{l}\text { Ploughman } \\
\text { et al. [19] }\end{array}$ & $\begin{array}{l}\text { Thanak- } \\
\text { wang } \\
\text { et al. }\end{array}$ & $\begin{array}{l}\text { Wallack } \\
\text { et al. [17] }\end{array}$ & $\begin{array}{l}\text { Robleda } \\
\text { et al. [18] }\end{array}$ & $\begin{array}{l}\text { Amosun } \\
\text { et al. [21] }\end{array}$ & $\begin{array}{l}\text { Lucas } \\
\text { et al. } \\
{[15]}\end{array}$ & Chen et al. [16] & $\begin{array}{l}\text { Hui Chian } \\
\text { Teh et al. } \\
\text { [20] }\end{array}$ \\
\hline \multicolumn{10}{|l|}{ Physical } \\
\hline Physical activity & N/A & N/A & $\checkmark$ & $\checkmark$ & $\checkmark$ & $\checkmark$ & $\checkmark$ & $\checkmark$ & $\checkmark$ \\
\hline Diet & N/A & N/A & $\checkmark$ & $\checkmark$ & & & $\checkmark$ & & \\
\hline \multicolumn{10}{|l|}{ Mental/cognitive } \\
\hline Self-awareness & $\checkmark$ & $\checkmark$ & & $\checkmark$ & & $\checkmark$ & $\checkmark$ & & \\
\hline Outlook/attitude & & $\checkmark$ & & $\checkmark$ & $\checkmark$ & $\checkmark$ & $\checkmark$ & $\checkmark$ & $\checkmark$ \\
\hline Life-long learning & & & $\checkmark$ & & $\checkmark$ & & & & $\checkmark$ \\
\hline Faith & & & $\checkmark$ & $\checkmark$ & $\checkmark$ & $\checkmark$ & $\checkmark$ & & \\
\hline \multicolumn{10}{|l|}{ Social } \\
\hline Social support & $\checkmark$ & $\checkmark$ & $\checkmark$ & $\checkmark$ & $\checkmark$ & & $\checkmark$ & & $\checkmark$ \\
\hline Financial security & & $\checkmark$ & $\checkmark$ & $\checkmark$ & $\checkmark$ & $\checkmark$ & & $\checkmark$ & $\checkmark$ \\
\hline Community engagement & & & $\checkmark$ & $\checkmark$ & $\checkmark$ & $\checkmark$ & $\checkmark$ & $\checkmark$ & $\checkmark$ \\
\hline \multicolumn{10}{|l|}{ Overlap } \\
\hline Independence & $\checkmark$ & $\checkmark$ & $\checkmark$ & $\checkmark$ & $\checkmark$ & & & & $\checkmark$ \\
\hline
\end{tabular}




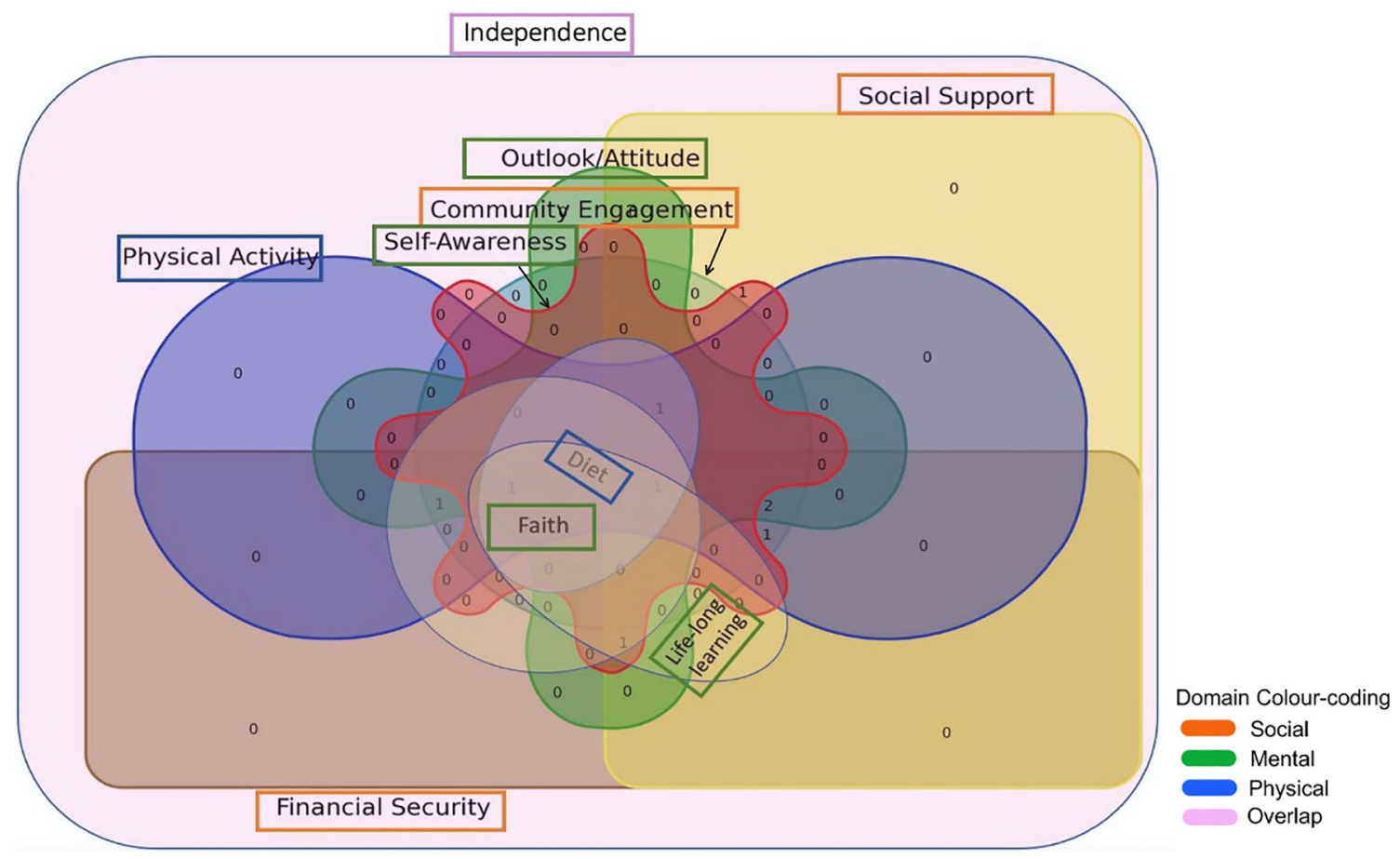

Fig. 2 Pictorial representation of determinants of healthy ageing. 0: no shared studies, 1: one shared study, 2: two shared studies. There are ten shapes, each representing a determinant. The border of the label of each shape is colour-coded according to the domain they correspond to. The numbers within each shape overlap represents

focused more on the aspect of exercise and keeping active as physical activity. Three studies used diet as a determinant for physical health, yet the specifics of the kind of diet or nutritional elements were not reported [14, 15, 17]. Lucas et al. included diet as part of the sustaining phase of healthy ageing due to its role in maintaining and supporting physical health [15].

\section{Mental/cognitive well-being}

All studies included mental/cognitive determinants of successful healthy ageing. Four main determinants emerged in relation to the mental/cognitive well-being domain, namely, self-awareness, outlook/attitude, life-long learning, and faith.

The determinant of self-awareness included self-esteem, self-achievement [13], resilience [19], body awareness, and sense of purpose [17]. Ploughman et al. defined resilience as "the participants ability to adapt to changes" specifically being conscious of the new circumstances they are presented with and choosing to modify their choices to support the new conditions [19]. This definition of resilience closely relates to Wallack et al. definition of body awareness, specifically relating to one's lifestyle choices [17]. Additionally, body how many studies included that combination of determinants. Venn diagram created using Bioinformatics and Evolutionary Genomics (http://bioinformatics.psb.ugent.be/cgi-bin/liste/Venn/calculate_venn. htpl)

awareness differs in the Wallack et al. study due to the specific circumstance of MS being studied [17].

The determinant of outlook/attitude, found in seven studies, ties into self-awareness [15-21]. Amosun et al. divided their findings into two overarching themes, one focused on participants found to have future-oriented behaviour and the second for participants without a future-oriented behaviour [21]. The final themes for successful ageing were specified within those who had a future-oriented behaviour, which included the theme of preparing for the afterlife. It was noted that having a good outlook and attitude towards the future impacted ageing in a positive way, rather than "awaiting death" [21].

Life-long learning (e.g. reading, taking up a new hobby, or learning a new language), found in three studies, is intricately connected with outlook/attitude [14, 18, 20]. Thanakwang et al. specifies that "engaging in active learning" is very important in successful healthy ageing particularly in the field of technology [14]. Additionally, continuous learning has a good cognitive impact aiding in maintaining one's cognitive function as they age.

Lastly, faith was found in five studies, which included the aspects of beliefs, religion, and spirituality [14, 15, 17, $18,21]$. Lucas et al. focused on incarcerated women as participants and created a framework that had the five stages of successful ageing [15]. Within the third phase ("reforming 
phase") and the fifth phase ("sustaining phase"), faith was significant [15]. Being in isolation has a large impact on mental health and immersing in faith was shown to support stability as well as increase motivation. Both of which support a good outlook towards life as the participants age and began to develop illnesses. Additionally, Robleda et al. found that participants reported that as you age it becomes more difficult to look forward to the future and immersing oneself in faith gave their life a higher sense of purpose [18].

\section{Social well-being}

All studies included social determinants of successful healthy ageing [13-21]. Three main determinants (Social Support, Financial Security, Community Engagement) were identified for the social domain.

Social support was reported across seven out of the nine studies [13-15, 17-20]. Social support was defined as establishing relationships and building rapport not only with family members but also with acquaintances. Additionally, Wallack et al. focused on MS patients, and brought up the factor of effective and accessible healthcare, which was classified as social support because participants' relationships with their care providers were valued [17].

Community engagement (identified in seven studies) ranged from volunteering to religious gatherings, such as going to church, and feeling acquainted with the community [14-18, 20, 21]. According to Amosun et al. engaging in community activities gave the participants a sense of purpose [21]. This was particularly explored by Hui Chian Teh et al. who focused on Chinese immigrants living in Australia [20].

The last determinant, which was identified across seven studies, was financial security [14, 16-21]. Robleda et al. defined financial security as being able to maintain a good quality of life [18], whereas Hui Chian Teh et al. focused on the aspect of not having to be a financial burden to family [20]. What both studies have in common was the emphasis on being able to maintain a good lifestyle; Hui Chian Teh et al. specified that having access and the ability to afford proper care as you age was highly important [20], which Wallack et al. agreed with for their MS participants [17]. The key aspect found across all studies that included financial security was the ability to continue to live a comfortable life and for many it included not having to rely on others.

\section{Independence as an overlap determinant}

Independence as a determinant was explored in six studies and it is present across all three domains [13, 14, 17-20]. It includes aspects, such as one's physical or mental/cognitive ability to live without support as well as being financially independent from family or friends. It was clearly shown in different studies that how independence is perceived changes according to the individual's circumstances. For Ploughman et al. and Wallack et al. both of whom focused on participants with MS, physical independence played a significant role in terms of how far their physical capability spanned $[17,19]$. The studies that did not research participants with MS also found independence to affect the physical domain as well as the social and mental/cognitive well-being domains. Due to the lack of a chronic disease, when independence was mentioned in these studies, it was not solely focused on the individual's physical independence. For Thanakwang et al. being self-reliant was a very important factor in the active ageing scale used [14].

\section{Discussion}

On 14th December 2020, the United Nations General Assembly declared 2021-2030 as the Decade of Healthy Ageing [22]. Healthy ageing replaced the WHO previous focus on active ageing. Although the concept of active healthy ageing has been widely researched and discussed in academic, political, and popular media arenas, systematic reviews that assess existing healthy ageing frameworks are lacking. To the best of our knowledge, this review illustrates the first attempt to systematically identify key determinants related to healthy ageing. The novelty of this research lies in the comparison of contemporary healthy ageing frameworks that have already been proposed. We identified ten determinants for healthy ageing, namely, physical activity, diet, self-awareness, outlook/attitude, life-long learning, faith, social support, financial security, community engagement, and independence.

The determinants of healthy ageing can vary depending on many factors, including culture, age, and gender. Therefore, it is important to consider that the studies were from varied geographical locations. This may have a large effect on what is considered important for achieving healthy ageing due to the difference in culture/customs [23]. Additionally, including a study with the premise of being an immigrant made it clear how integral community immersion and engagement is for an immigrant as they age, further emphasizing cultural differences. However, the geographical diversity arguably provided more depth and spread to this review, because it enabled the identification of commonalities, such as social support, independence, and financial security. This in turn will increase opportunities for local and global initiatives to optimise healthy ageing across different communities and countries.

Often, studies investigating healthy ageing focus on the biological factors (e.g. genetics and illnesses) that play a role in ageing [24]. We sought to identify modifiable factors to provide a better insight into healthy ageing. By doing this, non-biological factors, such as social, mental/cognitive, and physical well-being, were shown to play a substantial role [24]. For example, Wallack et al. who studied MS patients 
focused on the participants' acceptance and awareness of their body and its capability and how that largely impacted their mental health [17].

Our results illustrated that many of the determinants of physical, mental/cognitive, and social well-being are interrelated. For example, in the physical domain both determinants, physical activity and diet can affect the mental/cognitive determinant of attitude/outlook. Increasing physical activity and eating a balanced diet has been shown to boost the mood and energy levels of individuals which consequently improves their attitude/outlook towards life $[25,26]$. There was a contrast in terms of physical activity depending on the targeted group of participants, e.g. those with MS differed from those without. The inter-relation of determinants establishes the idea that healthy ageing cannot be segmented into isolated factors but is an inter-dependent measure. An example is how faith is linked to outlook/attitude, as it can be part of goal setting and gives individuals something to work on and improve as they age. Additionally, often, having a strong sense of faith aids an individual to find a greater sense of purpose. These inter-relations could be because different people place a higher value on different determinants, depending on their subjective views or life experiences [27]. Additionally, the inter-dependence between determinants supports the idea that healthy ageing is not a single stable measure, but that it is a balance that is constantly adjusted between all the determinants $[28,29]$. Therefore, to successfully evaluate healthy ageing there is a need to assess all the identified determinants and understand the value and hierarchy the individual ascribes to each determinant at the individual level. Independence could not be classified in only one domain since it has been found to be "highly significant for life satisfaction" and its loss to be a highly feared occurrence in ageing [30]. Thus, it was more appropriate to categorise it into an overlapping determinant included across all three domains.

This review gains its strengths from the combination of rigorous search and extraction methods and the underlying theoretical framework which guided the synthesis. Another strength of our work is that one of the exclusion criteria was studies that used clinical measurements for their results. This makes our proposed determinants more widely applicable to groups that do not have access to clinical diagnostic measures (e.g. blood tests, MRI). Additionally, by limiting the years of inclusion from 2010 to 2020, it was possible to focus on the most contemporary research available which builds on early established research in healthy ageing [28].

One of the limitations stems from the point of the original studies' definitions and categorisation. Most studies included in this review defined determinants differently, which made direct cross-cultural comparisons challenging. Only studies written in the English language were included, which might affect the ability to generalise results to non-Englishspeaking countries and may have resulted in us excluding relevant studies. Moreover, the studies included were cross sectional in nature, and therefore did not allow for investigation of causality between determinants and reports of healthy ageing. There was a larger proportion of female participants in the included studies, which might under-represent what males consider to be healthy ageing. The concept of healthy ageing is likely to be a dynamic process meaning important determinants may even vary within an individual depending on their age, further evaluation of relative contribution these determinants is warranted, albeit this is beyond the scope of the current study.

The application of the results from this review to preexisting longitudinal cohort data could provide direct comparison of these determinants in their contribution to healthy ageing at population level. Through our review we have created a more specialised understanding of healthy ageing by finding commonalities and differences among the nine identified frameworks. Future research would be to conduct a sense-checking exercise via focus group work with older adults to propose the new framework and whether this framework fits with their concept of healthy ageing. This is particularly important to evaluate whether all determinants have the same weighting towards defining healthy ageing and how it may vary with age, gender, race/ethnicity, and socioeconomic factors. Another alternative would be to cross reference this framework with large self-reported health studies to see how reliable and applicable these data are. Moreover, future studies should have an agreed terminology on how to better define determinants, which will be crucial for cross-cultural comparisons. Our results support the use of the term healthy ageing rather than successful or active ageing, in accordance with $\mathrm{Lu}$ et al. as it more holistically encompasses the domains of health as defined by the WHO [7, 8]. Additionally, going forward we suggest using the terms determinants rather than factors as it encompasses the direct effect that the determinants have on healthy ageing.

In summary, we have systematically reviewed the contemporary literature on frameworks of healthy ageing and identified ten determinants of successful healthy ageing. These are as follows: physical activity, diet, self-awareness, outlook/attitude, life-long learning, faith, social support, financial security, community engagement, and independence. Healthy ageing appears to be the result of all these determinants being optimised. By creating a clear framework of the factors that influence healthy ageing at an individual level, public service providers and policy makers can be guided to identify and give incentives to work towards improvement in health focusing on specific determinants that are relevant to an individual's circumstances.

Supplementary Information The online version contains supplementary material available at https://doi.org/10.1007/s40520-021-02049-w. 
Acknowledgements The authors would like to thank Dr Stavroula Lila Kastora for constructive criticism of the manuscript.

Author contributions TA and GK are joint first authors. KRM, KC, $\mathrm{MW}$, and PKM contributed to conceptualisation, funding acquisition, and supervision. TA and GK were involved in data curation, formal analysis, investigation, methodology, visualisation, and writing-original draft. All the authors contributed to writing-review and editing.

Funding TA and GK are recipients of The Autumn Project Research Scholarships (Ageing Clinical \& Experimental Research) funded by the Development Trust, University of Aberdeen. The authors acknowledge the donor of these scholarships, the Autumn Project Limited.

Data availability All data generated or analysed during this study are included in this article and its supplementary material files. Further enquiries can be directed to the corresponding author.

\section{Declarations}

Conflict of interest The authors declare no competing interests to declare.

Ethical approval An ethics statement was not required for this study type, no human or animal subjects or materials were used.

Statement of human and animal rights Not applicable.

Informed consent Not applicable.

\section{References}

1. The United Nations. World Population Ageing 2019: Highlights (online). Available at:https://www.un.org/en/development/desa/ population/publications/pdf/ageing/WorldPopulationAgeing2019Highlights.pdf. Accessed February 14, 2021.

2. Jowell A, Carstensen L, Barry M (2020) A life-course model for healthier ageing: lessons learned during the COVID-19 pandemic. Lancet Healthy Longev 1:e9-e10

3. Rowe J, Kahn R (1987) Human aging: usual and successful. Science 237:143-149

4. Hornby-Turner Y, Peel N, Hubbard R (2017) Health assets in older age: a systematic review. BMJ Open 7:013226

5. Menichetti J, Cipresso P, Bussolin D et al (2015) Engaging older people in healthy and active lifestyles: a systematic review. Ageing Soc 36:2036-2060

6. Lara J, Godfrey A, Evans E et al (2013) Towards measurement of the Healthy Ageing Phenotype in lifestyle-based intervention studies. Maturitas 76:189-199

7. Lu W, Pikhart H, Sacker A (2018) Domains and measurements of healthy aging in epidemiological studies: a review. Gerontologist 59:e294-e310

8. World Health Organisation. Constitution of the World Health (online). Available at: https://www.who.int/about/who-we-are/const itution. Accessed February 14, 2021

9. Moher D, Liberati A, Tetzlaff J et al (2009) Preferred reporting items for systematic reviews and meta-analyses: the PRISMA statement. PLoS Med 6:e1000097

10. Covidence - Better systematic review management. Covidence (online). Available at: https://www.covidence.org. Accessed November 2, 2020

11. Critical Appraisal Skills Programme. CASP Checklist (online). Available at: https://casp-uk.net/wp-content/uploads/2018/01/
CASP-Qualitative-Checklist-2018.pdf. Accessed November 17, 2020

12. Modesti P, Reboldi G, Cappuccio F et al (2016) Panethnic differences in blood pressure in Europe: a systematic review and metaanalysis. PLoS One 11:e0147601

13. Hyun Cha N, Ju Seo E, Sok S (2012) Factors influencing the successful aging of older Korean adults. Contemp Nurse 41:78-87

14. Thanakwang K, Isaramalai S, Hatthakit U (2014) Development and psychometric testing of the active aging scale for Thai adults. Clin Interv Aging. https://doi.org/10.2147/CIA.S66069

15. Lucas H, Lozano C, Valdez L et al (2018) A grounded theory of successful aging among select incarcerated older Filipino women. Arch Gerontol Geriatr 77:96-102

16. Chen L, Ye M, Kahana E (2019) A self-reliant umbrella: defining successful aging among the old-old (80+) in Shanghai. J Appl Gerontol 39:242-249

17. Wallack E, Wiseman H, Ploughman $M$ (2016) Healthy aging from the perspectives of 683 older people with multiple sclerosis. Mult Scler Int 2016:1-10

18. Robleda S, Pachana N (2017) Quality of life in Australian adults aged 50 years and over: data using the schedule for the evaluation of individual quality of life (SEIQOL-DW). Clin Gerontol 101:1-13

19. Ploughman M, Austin M, Murdoch M et al (2011) Factors influencing healthy aging with multiple sclerosis: a qualitative study. Disabil Rehabil 34:26-33

20. Hui Chian Teh J, Brown L, Bryant C (2019) Perspectives on successful ageing: the views of Chinese older adults living in Australia on what it means to age well. Australas J Ageing. https://doi.org/10. 1111/ajag. 12677

21. Amosun S, Harris F (2018) "What next now that you are sixty?"Preliminary exploration of the self-reported aspirations of community-dwelling older persons in the Western Cape Province, South Africa within the active aging framework. Physiother Theory Pract 36:791-798

22. World Health Organisation. Decade of Healthy Ageing (2021-2030) (online). Available at: https://www.who.int/initiatives/decade-ofhealthy-ageing. Accessed January 17, 2021.

23. Löckenhoff C, De Fruyt F, Terracciano A et al (2009) Perceptions of aging across 26 cultures and their culture-level associates. Psychol Aging 24:941-954

24. Reichstadt J, Depp C, Palinkas L et al (2007) Building blocks of successful aging: a focus group study of older adults' perceived contributors to successful aging. Am J Geriatr Psychiatry 15:194-201

25. Basso J, Suzuki W (2017) The effects of acute exercise on mood, cognition, neurophysiology, and neurochemical pathways: a review. Brain Plast 2:127-152

26. Huang Q, Liu H, Suzuki K et al (2019) Linking what we eat to our mood: a review of diet, dietary antioxidants, and depression. Antioxidants 8:376

27. Knight T, Ricciardelli L (2003) Successful aging: perceptions of adults aged between 70 and 101 Years. Int J Aging Hum Dev 56:223-245

28. Bowling A, Dieppe P (2005) What is successful ageing and who should define it? BMJ 331:1548-1551

29. Bryant L, Corbett K, Kutner J (2001) In their own words: a model of healthy aging. Soc Sci Med 53:927-941

30. Åberg A, Sidenvall B, Hepworth M et al (2005) On loss of activity and independence, adaptation improves life satisfaction in old age-a qualitative study of patients' perceptions. Qual Life Res 14:1111-1125

Publisher's Note Springer Nature remains neutral with regard to jurisdictional claims in published maps and institutional affiliations. 